\title{
Inactivation of INK4a and ARF induces myocardial proliferation and improves cardiac repair following ischemia-reperfusion
}

\author{
SONGTAO AN ${ }^{1}$, YAN CHEN ${ }^{1}$, CHUANYU GAO $^{1}$, BINGYU QIN $^{2}$, \\ XIANHUI DU ${ }^{2}$, FANMIN MENG $^{2}$ and YANYAN QI ${ }^{2}$ \\ Departments of ${ }^{1}$ Cardiology and ${ }^{2}$ Anesthesia, People's Hospital of Zhengzhou University, \\ Zhengzhou, Henan 450003, P.R. China
}

Received April 4, 2014; Accepted April 10, 2015

DOI: $10.3892 / \mathrm{mmr} .2015 .4133$

\begin{abstract}
The growth of the heart during mammalian embryonic development is primarily dependent on an increase in the number of cardiomyocytes (CM). However, shortly following birth, CMs cease proliferating and further growth of the myocardium is achieved via hypertrophic expansion of the existing CM population. The cyclin-dependent kinase inhibitor 2A (Cdkn2a) locus encodes overlapping genes for two tumor suppressor proteins, p16INK4a and p19 alternative reading frame (ARF). To determine whether decreased Cdkn2a gene expression results in improved cardiac regeneration in vitro and in vivo following cardiac injury, the proliferation of CMs isolated from Cdkn2a knockout (KO) and wild-type (WT) mice in vitro and in vivo were evaluated following generation of ischemia reperfusion (IR) injury. The KO mice demonstrated enhanced CM proliferation not only in vitro, but also in vivo. Furthermore, heart function was improved and scar size was decreased in the $\mathrm{KO}$ mice compared with that of the WT mice. The results also indicated that microRNA (miR)-1 and miR-195 expression levels associated with cell proliferation were reduced following IR injury in KO mice compared with those of WT mice. These results suggested that the inactivation of INK4a and ARF stimulated $\mathrm{CM}$ proliferation and promoted cardiac repair.
\end{abstract}

\section{Introduction}

Cardiovascular disease is the primary cause of human morbidity and mortality in developed countries (1). Despite progress in the development of clinical interventions to facilitate early myocardial reperfusion for patients with acute myocardial infarction (AMI), the rate of mortality during the acute phase

Correspondence to: Dr Yanyan Qi, Department of Anesthesia, People's Hospital of Zhengzhou University, 7 Weiwu Road, Zhengzhou, Henan 450003, P.R. China

E-mail: yyqi2010@163.com

Key words: cardiomyocyte, proliferation, ischemia-reperfusion, cyclin-dependent kinase inhibitor $2 \mathrm{~A}$ of AMI remains at $\sim 10 \%$ and the incidence of heart failure during the chronic phase reaches $25 \%(1,2)$. Although multiple methods of intervention have been shown to protect the heart from IR injury in experimental animals, almost no intervention has demonstrated clear protective effects against IR injury in a clinical setting (2). For this reason, significant resources have been focused on the elucidation of the molecular and pathophysiological characteristics of the diseased heart and vasculature, in order to aid the development of novel diagnostic and therapeutic strategies for cardiovascular disease $(1,2)$.

Amongst mammals, CM proliferation rapidly ceases following birth $(1,2)$. The transition of CMs from the proliferative state during embryonic development, to the differentiated, hypertrophic adult CM phenotype is tightly modulated $(1,2)$. Although there has been evidence of cardiac cell renewal amongst humans $(3,4)$, the proliferative capacity of adult CMs is limited. The ability of the adult heart to repair itself following injuries, including myocardial infarction or heart failure, is therefore significantly restricted (1).

However, a breakthrough was made, which demonstrated that the large T antigen of the SV40 virus induced the development of large tumors composed of dividing myocytes in the right atria of transgenic mice (5), and isolated myocytes (AT-1 cells) from these tumors were found to actively synthesize DNA and divide in culture (6,7). A study also revealed that myocytes isolated from transgenic tumors were able to proliferate in culture, whilst retaining the ability to contract spontaneously (8). These results indicated that the SV40 large $\mathrm{T}$ antigen may be able to induce terminally differentiated adult muscle cells to re-enter the cell cycle and divide again. $\mathrm{T}$ antigen oncoprotein is expressed at an early stage of cardiac development (6-8). Similarly to ATPase and helicase, $\mathrm{T}$ antigen interacts with specific proteins, including $\mathrm{pRb}, \mathrm{p} 53$ and p107, that are associated with the regulation of the cell cycle (9-11). Therefore, it was hypothesized that $\mathrm{T}$ antigen may function to prevent myocytes from exiting the cell cycle $(9,10)$.

As an oncogene, retinoblastoma protein $(\mathrm{Rb})$ is involved in the orchestration of mitotic arrest and prevention of cell cycle re-entry, as well as the inhibition of apoptosis and regulation of stable tissue-specific gene expression (12). It was also shown that, in combination with suppression of alternative reading frame (ARF), the suppression of $\mathrm{Rb}$ reversed differentiation and postmitotic arrest in skeletal muscle cells (13). However, 
the differentiation of cardiac muscle is in contrast to that of skeletal muscles, in which DNA synthesis and the synthesis of differentiated cell products and contractile proteins do not occur simultaneously (13). Multiple studies have also suggested that the adult heart retains limited regenerative capacity via an additional pathway, comprising activation of tyrosine kinase receptors ErbB2/4 in response to treatment with neuregulin-1 $(14,15)$. These findings suggested that the regulation of certain genes and proteins may stimulate the proliferation of adult CMs, challenging current views of the heart as a postmitotic organ (16).

MicroRNA (miRNAs) are a class of highly conserved, single-stranded, small non-coding RNAs, which were identified as transcriptional and post-transcriptional inhibitors of gene expression (17). These miRNAs 'fine tune' the translational output of target messenger RNAs $(17,18)$. Certain miRNAs, including miR-1 $(19,20)$ and miR-195 (21), have been shown to inhibit CM proliferation.

The present study aimed to elucidate the functions of Cdkn2a in CM differentiation and potential re-entry to the cell cycle in vitro and in vivo following cardiac injury. The proliferation of CMs isolated from Cdkn2a (p16p19) knockout (KO) and wild-type (WT) mice in vitro as well as that in tissue from in vivo experiments following ischemia/reperfusion (IR) injury was therefore analyzed. As miRNAs had previously been identified as critical regulators of gene expression in response to IR (22), miR-1 and miR-195 expression following inactivation of the $\mathrm{Cdkn} 2 \mathrm{a}$ gene were also evaluated.

\section{Materials and methods}

Animals. All animal procedures were performed according to regulation policy approved by China Animal Care Committees. The homozygous Cdkn2a KO mouse strain FVB.129-Cdkn2a $\mathrm{a}^{\mathrm{tm} 1 \mathrm{Rdp}}$ with an FVB/n genetic background was obtained from the National Resource Center for Mutant Mice (Nanjing, China). This strain carries a targeted deletion of exons 2 and 3 of the INK4a/ARF locus, which eliminates p16 and p19. WT littermate mice were used as controls for all experiments. The mice (weighing 40-60 g; 8 weeks-old) underwent a $12 \mathrm{~h}$ light/dark cycle, with ad libitum access to food and water. The mice were maintained at room temperature in an atmosphere containing $60 \%$ humidity. The present study was approved by the ethics committee of Zhengzhou University (Zhengzhou, China).

Isolation and culture of CMs. CMs were isolated as previously described (23). Briefly, 8-10 week-old mice were anesthetized by interperitoneal injection of isoflurane (Roche Diagnostics, Basel, Switzerland) with heparin (100 units isoflurane/mouse). Subsequently, the heart was removed from the chest, cannulated via the ascending aorta and mounted on a modified perfusion system apparatus (IFB-01; SKE Advanced Therapies, Milan, Italy). Following perfusion for $4 \mathrm{~min}$ at $3 \mathrm{ml} / \mathrm{min}$, enzyme solution $(0.25 \%$ trypsin $)$ containing collagenase type II (Worthington, Lakewood, NJ, USA) supplemented with $100 \mathrm{mM} \mathrm{CaCl}{ }_{2}$ was used for another perfusion for $\sim 8-10 \mathrm{~min}$. The ventricles were cut into smaller sections with forceps, triturated, transferred to $15-\mathrm{ml}$ conical tubes and centrifuged at $500 \mathrm{x}$ g for $3 \mathrm{~min}$. Subsequently, the calcium concentration was adjusted to $0.5 \mathrm{mM}$ with $100 \mathrm{mM} \mathrm{CaCl}_{2}$. The cells were plated in petri dishes coated with Laminin (Invitrogen Life Technologies, Carlsabad, CA, USA). Dulbecco's modified Eagle's medium (DMEM; Hyclone, GE Healthcare Life Sciences, Logan UT, USA) was added to facilitate continued cell growth $1 \mathrm{~h}$ following plating.

Closed-chest myocardial infarction procedure. IR was surgically induced as described previously (24). The mice were anesthetized by intravenous injection of pelltobarbitalum (0.5 mg/kg, Sigma-Aldrich, Carlsbad, CA, USA). Briefly, the closed-chest left anterior descending coronary artery occlusion model of IR was used (45 min ischemia) and sham animals were subjected to identical surgical procedures, without coronary occlusion.

Immunofluorescent and immunohistological analyses. CMs were fixed in 4\% paraformaldehyde (PFA; Sigma-Aldrich, St. Louis, MO, USA), washed in phosphate-buffered saline (PBS; Sigma-Aldrich), incubated cells with primary antibodies at $4^{\circ} \mathrm{C}$ overnight and then incubated with secondary antibodies for labeling. Cells were mounted with DAPI $(0.4 \mu \mathrm{g} / \mathrm{ml}$; cat no. H-1200; Vector Laboratories Inc., Burlingame, CA, USA) in DMEM and viewed with a Leica-TCS SP5 confocal microscope (Leica Microsystems GmbH, Wetzlar, Germany).

Heart tissues were harvested at various time-points (days 2 and 30) following MI, fixed with $4 \%$ PFA at $4^{\circ} \mathrm{C}$ overnight and subsequently with $20 \%$ sucrose at $4^{\circ} \mathrm{C}$ overnight. Optimum cutting temperature compound (Sigma-Aldrich) was used to freeze the samples. The slides were incubated with primary antibodies at $4^{\circ} \mathrm{C}$ overnight and then incubated with secondary antibodies for labeling. Slides were mounted with DAPI (Vector Laboratories, Inc.), and all images were acquired using a Leica-TCS SP5 confocal microscope (Leica Microsystems $\mathrm{GmbH}$ ).

The primary antibodies used were as follows: Rabbit polyclonal anti-mouse proliferating cell nuclear antigen (PCNA) antibody (1:2,000; ab2426; Abcam, Cambridge, UK), mouse monoclonal immunoglobulin $\mathrm{G}_{1}$ cardiac troponin $\mathrm{T}-\mathrm{cTnT}$ (clone 13-11; Thermo Fisher Scientific, Waltham, MA, USA) and rabbit polyclonal anti-Ki67 antibody (1:2,000; ab15580; Abcam). Goat anti-rabbit and anti-mouse secondary antibodies were coupled with Alexa 488, Alexa 555 or Alexa 633 (1:3,000; Invitrogen Life Technologies).

Reverse transcription-quantitative polymerase chain reaction (RT-qPCR) analysis of miRNA expression. RNA was purified using an miRNAease Mini Purification kit (Cat no. 217004; Qiagen, Hilden, Germany), cDNA synthesis was performed with a cDNA synthesis kit (cat no. G269; Applied Materials Inc., Santa Clara, CA, USA) and RT-qPCR was performed using an EvaGreen miRNA qPCR Mastermix-ROX kit (cat ID, Mastermix-ms; Applied Material Development, Inc.), according to the manufacturer's instructions and using Eppendorf $^{\circledR}$ Realplex 2 miRNA primers: miR-1 (cat no., MPM00015) and miR-195 (cat no., MPM00177), purchased from Applied Material Development, Inc.

Echocardiography analysis. Two and 30 days following IR, transthoracic echocardiography was performed using 


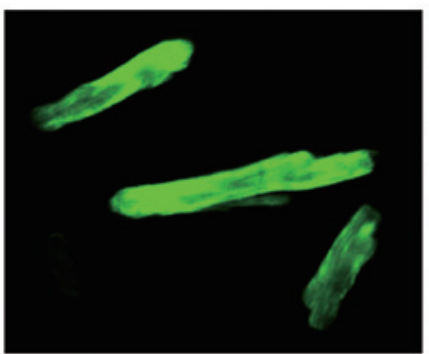

KO cTnT

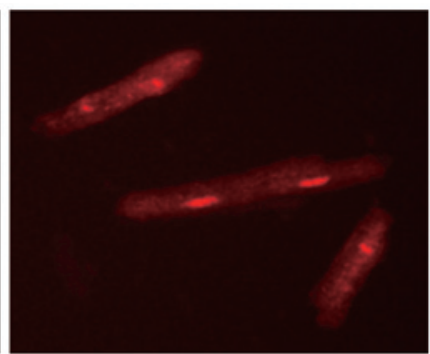

KO PCNA
Figure 1. Analysis of proliferation of $\mathrm{CMs}$ isolated from $\mathrm{KO}$ mice were cultured in vitro for two days for labeling of PCNA and cTnT. PCNA (red), proliferation marker; cTnT (green), CM-specific marker (magnification x200). KO, cyclin-dependent kinase inhibitor 2A knockout; cTnT, cardiac troponin T; PCNA, proliferating cell nuclear antigen; CM, cardiomyocyte.

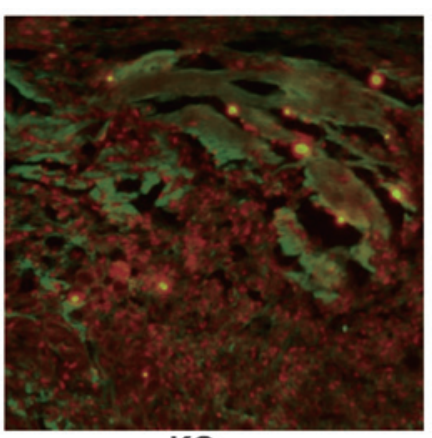

KO

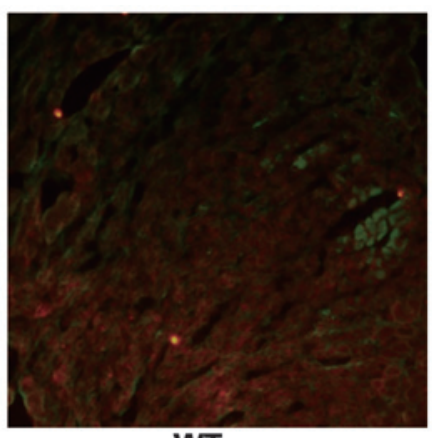

WT
Figure 2. Analysis of proliferation in the infarct region of KO and WT mice in vivo following four days of reperfusion. cTnT/PCNA proliferation analysis was performed following four days of reperfusion in KO and WT mice. cTnT (green), CM-specifc marker; PCNA (red), proliferation marker. There were $\sim 60-70 \%$ CMs exhibiting positive PCNA labeling in KO mice following ischemia/reperfusion, but low levels in WT mice (magnification, $\mathrm{x} 200$ ). KO cyclin-dependent kinase inhibitor 2A knockout; WT, wild-type; cTnT, cardiac troponin T; PCNA, proliferating cell nuclear antigen; CM, cardiomyocyte.

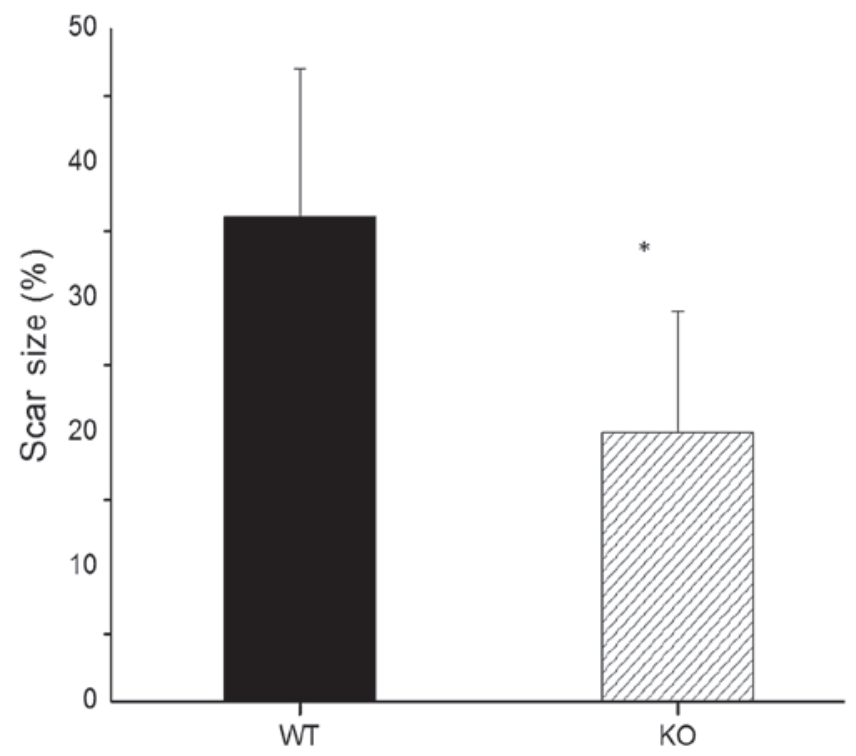

Figure 3. Analysis of scar size following 30 days of reperfusion in KO and WT mice. Scar sizes were analyzed at day 30 following ischemia/reperfusion in KO and WT mice. Scar size in KO mice was smaller than that in WT mice. Values are presented as the mean \pm standard error of the mean. $\mathrm{KO}, \mathrm{n}=9$ and WT, $\mathrm{n}=11 .{ }^{*} \mathrm{P}<0.01$ vs. WT. KO, cyclin-dependent kinase inhibitor $2 \mathrm{~A}$ knockout; WT, wild-type. a cardiovascular ultrasound system (Vivid 7; General Electric Co., Fairfield, CT, USA) with a 10-MHz linear-array transducer. Animals were weighed and anesthetized with $1.5 \%$ isoflurane and placed on a warm blanket. Cardiac long-axis and short-axis views were obtained in the two-dimensional mode and M-mode tracings were recorded. Left ventricle (LV) end-systolic dimension (LVESD), LV end-diastolic dimension (LVEDD), interventricular septal thickness in the diastole (IVSd) and LV posterior wall thickness (LVPW), analyzed in the M-mode tracings at the papillary muscle level, were measured in a minimum of three consecutive cardiac cycles. Percent LV fractional shortening (\%FS) was calculated as: $\%$ FS $=($ LVEDD-LVESD $) /$ LVEDDx100. Researchers who performed echocardiograms and measured echocardiographic parameters were blinded to the identity of $\mathrm{KO}$ mice among groups. Echocardiographic parameters were re-measured by an additional blinded researcher and found to be consistent.

Analysis of scar size post-MI. Scar size was measured 30 days following IR using a protocol based on a previously described method (25). Briefly, mouse hearts were arrested in the diastole with saturated $\mathrm{KCl}$ (2 ml, $1 \mathrm{~mol} / \mathrm{l}$; Tiangen Biotech Co., Ltd., Beijing, China), which was injected into the left ventricular chamber, removed, embedded in O.C.T. compound and frozen. Hearts were subsequently sliced transversely to a $10-\mu \mathrm{m}$ thickness with $300 \mu \mathrm{m}$ between sections. Sections from the apex to the base of the ventricle were stained with Masson trichrome (Wuhan Boshide Biological Engineering Co., Ltd., Wuhan, China) to determine infarct size with the midline arc length method $(15,25)$. Sections were scored blindly to evaluate the extent of fibrosis (15).

Statistical analysis. Values are presented as the mean \pm standard error. For the comparison of infarct size and miRNA expression between the KO and WT groups, the independent-samples t-test was used. For the comparison of LVFS, LVEDD and LVESD on days two and 30 between the KO and WT mice, a two-way analysis of variance with Tukey's post hoc test was used. $\mathrm{P}<0.05$ was considered to indicate a statistically significant difference. Statistical analyses were performed using SPSS version 19.0 (International Business Machines, Armonk, NY, USA).

\section{Results}

CM proliferation is induced in Cdkn2a KO mice. To evaluate whether the inactivation of p16INK4a and p19ARF influenced adult mouse CM proliferation, CMs isolated from $\mathrm{KO}$ and WT mice were cultured for 1, 2 and 3 days prior to the detection of proliferation marker PCNA labeling. As indicated in Fig. 1, 60-70\% CMs were positively labelled for PCNA in the KO mouse. There were no significant differences detected between the cells cultured for 1,2 and 3 days. However, less PCNA-positive CMs were isolated from WT mice.

CMs in infarct region of $\mathrm{KO}$ mice demonstrate enhanced proliferation. To detemine whether the $\mathrm{Cdkn} 2 \mathrm{a}$ deletion influenced $\mathrm{CM}$ proliferation in the cardiac injury region and improved recovery following cardiac injury, IR was induced in $\mathrm{KO}$ and 

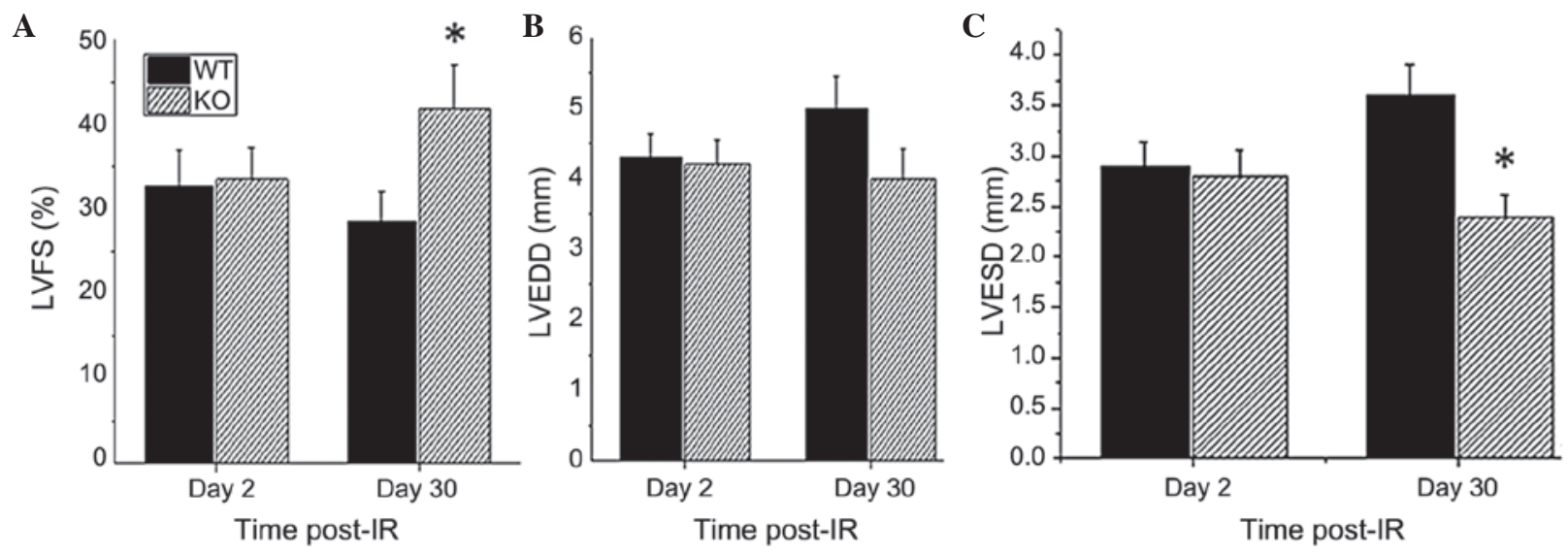

Figure 4. Evaluation of heart function by echocardiography at baseline on days two and 30 post-IR (A) LVFS, (B) LVEDD and (C) LVESD. Values are expressed as the mean \pm standard error of the mean. KO, $n=9$ and WT, $n=11 .{ }^{*} \mathrm{P}<0.01 \mathrm{vs}$. WT. LVFS, percentage of LV fractional shortening; IR, ischemia/reperfusion; LVESD, left ventricle end-systolic dimension; LVEDD, left ventricle end-diastolic dimension; WT, wild-type; KO, cyclin-dependent kinase inhibitor $2 \mathrm{~A}$ knockout.

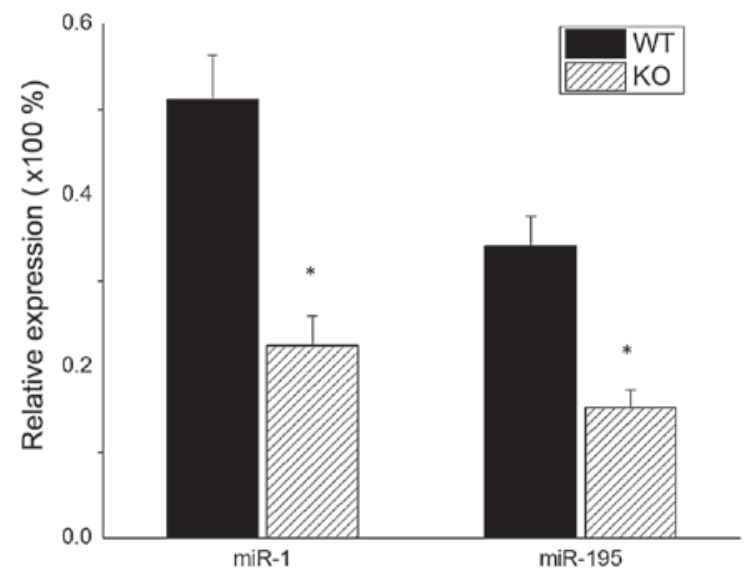

Figure 5. miRNA-1 and -195 expression following two days of reperfusion in KO and WT mice. RNA was purified from the infarct and normal regions of $\mathrm{KO}$ and $\mathrm{WT}$ mice following two days of reperfusion and used to synthesize complementary DNA. RT-qPCR results in the infarction region are displayed as the percentage of levels in the normal region. miR-1 and -195 levels in the infarction region in KO mice were lower than those in WT mice. Values are presented as the mean \pm standard error of the mean $\mathrm{n}=5$, per group. ${ }^{*} \mathrm{P}<0.01$ vs. WT. WT, wild-type; KO, cyclin-dependent kinase inhibitor $2 \mathrm{~A}$ knockout; RT-qPCR, reverse transcription-quantitative polymerase chain reaction; miRNA, microRNA.

WT mice. PCNA labeling was analyzed following four and 30 days of reperfusion, respectively. PCNA-positive CMs were detected in KO mice, whereas none were detected in WT mice. Of note, in KO mice, more PCNA-positive cardiomyocytes were detected following four days of reperfusion than following 30 days of reperfusion (Fig. 2). However, PCNA-positive CMs were still detected following 30 days of reperfusion (data not shown).

Scar size as well as ventricular wall and heart function are improved in KO mice at day 30 post-IR. To determine whether there were alterations in heart morphology and/or improvements in heart function, infarct scar size and heart function were measured in $\mathrm{KO}$ and WT mice 30 days following IR injury. The results (Fig. 3) indicated that scar size was significantly reduced in KO mice $(n=9)$ compared with that in WT mice $(\mathrm{n}=10)(\mathrm{P}<0.01)$.
Left ventricular FS\% was measured by echocardiography to evaluate heart function. There was no significant difference in the FS\% between KO and WT mice following two days of reperfusion. FS\% following 30 days of reperfusion in KO mice was significantly greater than that in WT mice (Fig. 4A). Following 30 days of reperfusion, FS\% was improved compared to that at day two post-IR in KO mice, but was significantly reduced in WT mice (Fig. 4A). The LVEDD level in $\mathrm{KO}$ mice was significantly lower, as compared with in the WT mice (Fig. 4B). Furthermore, the LVESD level in the KO mice was also significantly lower, as compared with in the WT mice (Fig. 4C).

miR-1 and miR-195 expression is downregulated in KO mice. To evaluate whether miR-1 and miR-195 expression levels were altered in the infarct region, RNA from the infarction and a remote region were analyzed for miR-1 and -195 expression using RT-qPCR. As shown in Fig. 5, the results indicated that miR-1 levels on day two post-IR were decreased in the infarction region in KO and WT mice as compared with those in normal regions. However, miR-1 and miR-195 levels were significantly downregulated in KO mice compared with those in WT mice.

\section{Discussion}

To the best of our knowledge, the present study was the first to demonstrate that CM proliferation in vitro and in vivo may be stimulated by inactivation of the Cdkn2a gene, and that inactivation of Cdkn2a was able to promote functional recovery following IR injury in adult animals.

The Cdkn2a gene is stimulated following DNA damage and has a significant role in the prevention of aberrant cellular proliferation (26). It was previously hypothesized that inactivation of Cdkn2a and ARF gene expression may promote tissue regeneration by inducing an increase in stem cell self-renewal $(27,28)$, decreasing phenotype-associated senescence, including enhancing the production of fibrosis-inducing factors by fibroblasts and epithelial cells (29), and the induction of dedifferentiation and proliferation of somatic cells following injury $(13,30)$. 
In the present study, it was demonstrated that the inactivation of Cdkn2a influenced adult mouse CM proliferation in vivo and in vitro, and improved recovery following IR-induced cardiac injury. The results revealed that $60-70 \%$ of CMs isolated from adult $\mathrm{KO}$ mice were positively labeled for PCNA at culture days one, two and three. However, no PCNA-positive CMs were isolated from WT mice. The results of the present study also indicated that following 30 days of reperfusion, heart function was improved and scar size was reduced in $\mathrm{KO}$ mice compared with that in WT mice.

To date, the molecular mechanisms underlying CM cell cycle exit and permanent growth arrest have remained elusive. $\mathrm{Rb}$ has previously been implicated in regulating cell cycle exit, as well as the irreversibility of cell cycle arrest in skeletal muscle $(13,31)$. Rb knockout induced fibroblasts to transdifferentiate into skeletal myoblasts in vitro $(32,33)$. The deletion of $\mathrm{Rb}$ during differentiation results in the formation of normal multinucleated myotubes; therefore, $\mathrm{Rb}$ appears to be uniquely required for normal cell cycle control and differentiation (34). CMs from cardiac-restricted Rb-deficient mice develop normally and do not exhibit defects in the cardiac cell cycle (35). These observations therefore suggest that $\mathrm{Rb}$ and $\mathrm{p} 130$, or $\mathrm{Rb}$ together with other genes, may have critical roles in the regulation of cardiac myocyte cell cycle exit $(34,35)$. In agreement with the results of the present study, previous studies have indicated that the inactivation of $\mathrm{Rb}$ and ARF lead to mammalian muscle cell cycle re-entry, loss of differentiation properties and upregulation of cytokinetic machinery (13).

A study has also indicated that Erb2 may be involved in the regulation of CM proliferation (14). Injection of cardiac progenitor cells into the infarct zone on day three post-MI reduced scar size and improved heart function at day $30(36,37)$. The results of the present study indicated that inactivation of $\mathrm{Cdkn} 2 \mathrm{a}$ promoted $\mathrm{CM}$ proliferation in the infarct region post-MI, subsequently enhancing cardiac muscle regeneration to improve heart function following cardiac injury.

Increasing evidence has indicated that miRNAs are able to regulate cellular function, including cell proliferation, migration, differentiation and apoptosis $(38,39)$.

The results of the present study indicated that INK4a and ARF elimination improved heart function by enhancing myocardial regeneration via the regulation of miRNA expression, including miR-1 and -195. , miR-1 has been suggested to modulate the balance between the levels of differentiation and proliferation of cardiac precursor cells during cardiogenesis by targeting Hand2, a transcription factor which promotes the expansion of ventricular CMs $(19,20)$. miR-195 is part of the miR-15 family, which comprises miR-15a, miR-15b, miR-16-1, miR-16-2, miR-424 and miR-497. A study previously indicated that miR-15 family members inhibit cell proliferation (21).

The present study demonstrated that the reduced expression levels of miR-1 and miR-195 in cyclin-dependent kinase inhibitor $2 \mathrm{~A} \mathrm{KO}$ mice may aid the enhanced $\mathrm{CM}$ proliferation. However, further study is required in order to verify this function.

The results of the present study, evaluating the INK4a/ARF deletion, did not differentiate between the effects of p16 and p19, and therefore the results may be perturbed by the pleiotropic effects of the gene knockout. Future experiments utilizing p16- and p19-selective $\mathrm{KO}$ mice are required to provide definitive evidence regarding whether the regenerative effects observed are mainly mediated by one or the other of these genes.

Regeneration of cardiac mass appears to be significant in numerous pathological conditions associated with CM loss, including MI, heart failure and dilated cardiomyopathy $(40,41)$. Therefore, the observation that deletion of the Cdkn2a gene has the potential to improve cardiac function following MI injury is of significant translational value. Specific miRNAs to inhibit Cdkn2a RNA gene expression may be developed in order to improve $\mathrm{CM}$ regeneration for the treatment of multiple types of cardiac injury.

\section{Acknowledgements}

The authors would like to thank Dr Jianjun Yuan (People's Hospital of Zhengzhou University) for providing excellent technical assistance. The present study was supported by the Department of Science and Technology of Henan Province. Grants were provided by the Outstanding Youth Fund of Zhengzhou University.

\section{References}

1. Ahuja P, Sdek P and MacLellan WR: Cardiac myocyte cell cycle control in development, disease, and regeneration. Physiol Rev 87: 521-544, 2007.

2. van Amerongen MJ and Engel FB: Features of cardiomyocyte proliferation and its potential for cardiac regeneration. J Cell Mol Med 12: 2233-2244, 2008.

3. Bergmann O, Bhardwaj RD, Bernard S, et al: Evidence for cardiomyocyte renewal in humans. Science 324: 98-102, 2009.

4. Kajstura J, Urbanek K, Perl S, et al: Cardiomyogenesis in the adult human heart. Circ Res 107: 305-315, 2010.

5. Field LJ: Atrial natriuretic factor-SV40 T antigen transgenes produce tumors and cardiac arrhythmias in mice. Science 239: 1029-1033, 1988.

6. Delcarpio JB, Lanson NA Jr, Field LJ and Claycomb WC: Morphological characterization of cardiomyocytes isolated from a transplantable cardiac tumor derived from transgenic mouse atria (AT-1 cells). Circ Res 69: 1591-1600, 1991.

7. Steinhelper ME, Lanson NA Jr, Dresdner KP, et al: Proliferation in vivo and in culture of differentiated adult atrial cardiomyocytes from transgenic mice. Am J Physiol 259: H1826-H1834, 1990.

8. Katz EB, Steinhelper ME, Delcarpio JB, Daud AI, Claycomb WC and Field LJ: Cardiomyocyte proliferation in mice expressing alpha-cardiac myosin heavy chain-SV40 T-antigen transgenes. Am J Physiol 262: H1867-H1876, 1992.

9. Fanning E: Simian virus 40 large T antigen: the puzzle, the pieces, and the emerging picture. J Virol 66: 1289-1293, 1992.

10. Lewin B: Driving the cell cycle: $M$ phase kinase, its partners, and substrates. Cell 61: 743-752, 1990.

11. Weinberg RA: Tumor suppressor genes. Science 254: 1138-1146, 1991.

12. Burkhart DL and Sage J: Cellular mechanisms of tumour suppression by the retinoblastoma gene. Nat Rev Cancer 8: 671-682, 2008.

13. Pajcini KV, Corbel SY, Sage J, Pomerantz JH and Blau HM: Transient inactivation of $\mathrm{Rb}$ and ARF yields regenerative cells from postmitotic mammalian muscle. Cell Stem Cell 7: 198-213, 2010.

14. Pentassuglia L and Sawyer DB: The role of Neuregulin-1beta/ErbB signaling in the heart. Exp Cell Res 315: 627-637, 2009.

15. Bersell K, Arab S, Haring B and Kuhn B: Neuregulin1/ErbB4 signaling induces cardiomyocyte proliferation and repair of heart injury. Cell 138: 257-270, 2009.

16. Braun T and Dimmeler S: Breaking the silence: stimulating proliferation of adult cardiomyocytes. Dev Cell 17: 151-153, 2009. 
17. Bartel DP: MicroRNAs: genomics, biogenesis, mechanism, and function. Cell 116: 281-297, 2004.

18. Lee RC, Feinbaum RL and Ambros V: The C. elegans heterochronic gene lin-4 encodes small RNAs with antisense complementarity to lin-14. Cell 75: 843-854, 1993.

19. Zhao Y, Samal E and Srivastava D: Serum response factor regulates a muscle-specific microRNA that targets Hand2 during cardiogenesis. Nature 436: 214-220, 2005.

20. Zhao Y, Ransom JF, Li A, et al: Dysregulation of cardiogenesis, cardiac conduction, and cell cycle in mice lacking miRNA-1-2 Cell 129: 303-317, 2007.

21. Porrello ER, Johnson BA, Aurora AB, et al: MiR-15 family regulates postnatal mitotic arrest of cardiomyocytes. Circ Res 109: 670-679, 2011

22. Ren XP, Wu J, Wang X, et al: MicroRNA-320 is involved in the regulation of cardiac ischemia/reperfusion injury by targeting heat-shock protein 20. Circulation 119: 2357-2366, 2009.

23. Kabaeva Z, Zhao M and Michele DE: Blebbistatin extends culture life of adult mouse cardiac myocytes and allows efficient and stable transgene expression. Am J Physiol Heart Circ Physiol 294: H1667-H1674, 2008.

24. Nossuli TO, Lakshminarayanan V, Baumgarten G, et al: A chronic mouse model of myocardial ischemia-reperfusion: essential in cytokine studies. Am J Physiol Heart Circ Physiol 278: H1049-H1055, 2000.

25. Takagawa J, Zhang Y, Wong ML, et al: Myocardial infarct size measurement in the mouse chronic infarction model: comparison of area- and length-based approaches. J Appl Physiol 102: 2104-2111, 2007.

26. Serrano M, Lee H, Chin L, Cordon-Cardo C, Beach D and DePinho RA: Role of the INK4a locus in tumor suppression and cell mortality. Cell 85: 27-37, 1996.

27. Lewis JL, Chinswangwatanakul W, Zheng B, et al: The influence of INK4 proteins on growth and self-renewal kinetics of hematopoietic progenitor cells. Blood 97: 2604-2610, 2001.

28. Zhang HW, Ding J, Jin JL, et al: Defects in mesenchymal stem cell self-renewal and cell fate determination lead to an osteopenic phenotype in Bmi-1 null mice. J Bone Miner Res 25: 640-652, 2010.

29. Campisi J and d'Adda di Fagagna F: Cellular senescence: when bad things happen to good cells. Nat Rev Mol Cell Biol 8: 729-740, 2007.
30. Lee DH, Wolstein JM, Pudasaini B and Plotkin M: INK4a deletion results in improved kidney regeneration and decreased capillary rarefaction after ischemia-reperfusion injury. Am J Physiol Renal Physiol 302: F183-F191, 2012.

31. Gu W, Schneider JW, Condorelli G, Kaushal S, Mahdavi V and Nadal-Ginard B: Interaction of myogenic factors and the retinoblastoma protein mediates muscle cell commitment and differentiation. Cell 72: 309-324, 1993.

32. Novitch BG, Mulligan GJ, Jacks T and Lassar AB: Skeletal muscle cells lacking the retinoblastoma protein display defects in muscle gene expression and accumulate in $\mathrm{S}$ and $\mathrm{G} 2$ phases of the cell cycle. J Cell Biol 135: 441-456, 1996.

33. Novitch BG, Spicer DB, Kim PS, Cheung WL and Lassar AB: $\mathrm{pRb}$ is required for MEF2-dependent gene expression as well as cell-cycle arrest during skeletal muscle differentiation. Curr Biol 9: 449-459, 1999.

34. Huh MS, Parker MH, Scime A, Parks R and Rudnicki MA: Rb is required for progression through myogenic differentiation but not maintenance of terminal differentiation. J Cell Biol 166: 865-876, 2004

35. MacLellan WR, Garcia A, Oh $\mathrm{H}$, et al: Overlapping roles of pocket proteins in the myocardium are unmasked by germ line deletion of p130 plus heart-specific deletion of Rb. Mol Cell Biol 25: 2486-2497, 2005.

36. Smart N, Bollini S, Dubé KN, et al: De novo cardiomyocytes from within the activated adult heart after injury. Nature 474: 640-644, 2011

37. Ye J, Boyle A, Shih H, et al: Sca-1+ cardiosphere-derived cells are enriched for Isl1-expressing cardiac precursors and improve cardiac function after myocardial injury. PLoS One 7: e30329, 2012.

38. Tang Y, Zheng J, Sun Y, Wu Z, Liu Z and Huang G: MicroRNA-1 regulates cardiomyocyte apoptosis by targeting Bcl-2. Int Heart J 50: 377-387, 2009.

39. Frost RJ and van Rooij E: miRNAs as therapeutic targets in ischemic heart disease. J Cardiovasc Transl Res 3: 280-289, 2010.

40. Dorn GW II: Apoptotic and non-apoptotic programmed cardiomyocyte death in ventricular remodelling. Cardiovasc Res 81: 465-473, 2009

41. Nishida K and Otsu K: Cell death in heart failure. Circ J 72 (Suppl A): A17-A21, 2008. 